Saggio scientifico originale

CDU 821.131.1.09 Alighieri, D.

821.131.1:821.163.42

\title{
L'INFLUSSO DELLE OPERE DI DANTE ALIGHIERI SULLA LETTERATURA E LA CULTURA CROATE
}

\author{
Eliana Moscarda Mirković \\ Ivana Perišić \\ Università Juraj Dobrila di Pola \\ Dipartimento di studi in lingua italiana
}

\section{Riassunto}

Il presente saggio espone i risultati di una ricerca storico-letteraria riguardante I'influenza delle opere di Dante Alighieri sulla produzione e sulla traduzione letteraria croate. L'intento è quello di porre in rilievo l'importanza dell'influsso di Dante in Croazia, anche in considerazione del fatto che non pochi, oggigiorno, sembrano essere all'oscuro del fatto che il grande poeta-vate ebbe a soggiornare nei nostri territori, rimanendone così affascinato da volerne fare esplicita menzione nel proprio capolavoro - la Divina Commedia. Seguendo le tracce della sua fortuna in terra croata, scopriremo in tal modo che Dante Alighieri non solo ha reso possibile lo sviluppo della lingua volgare italiana, ma ha anche dato un significativo impulso allo sviluppo della lingua croata e, attraverso le traduzioni di Kombol e Kršnjavi, ha contribuito all'integrazione della Croazia nel panorama letterario europeo.

Parole chiave: Dante Alighieri, Divina Commedia, letteratura croata, Kombol, Markuš, Kršnjavi 


\section{Dante nella letteratura croata. Le traduzioni in croato della Divina Commedia}

Ciò che rende speciale una lingua è il suo registro scritto, cioè la letteratura. La varietà parlata è stata da sempre quella più facile da apprendere, ma la letteratura, attraverso quei testi che possiamo definire classici, ha ben presto trovato il proprio posto accanto alla lingua parlata ed ha contribuito a consolidarla. Ogni continente, ogni Paese ha i propri classici, che fanno parte della storia e della cultura nazionale e servono come base per lo sviluppo di altre forme scritte.

La necessità di confrontarsi coi classici della letteratura mondiale si è fatta sentire, in Croazia, non unicamente come modalità di ampliamento del corpus letterario nazionale, ma anche e soprattutto al fine di dimostrare che la lingua croata è all'altezza di tutti i moduli espressivi tipici delle lingue di cultura, ed è pertanto perfettamente adeguata a tradurre i grandi nomi della letteratura internazionale.

Fino alla metà del XX secolo, in Croazia non esistevano traduzioni integrali della Divina Commedia, o meglio ne esistevano traduzioni o tentativi di traduzione, che non si erano tuttavia rivelati all'altezza delle attese in essi riposte. Già dalla seconda metà del XV secolo, infatti, si registrano tentativi, da parte di alcuni letterati croati, di trasporre la Divina Commedia (come pure altri classici della letteratura mondiale) nel codice linguistico croato. ${ }^{1}$ Gli autori-traduttori croati dell'epoca furono ben inseriti nel pensiero e nelle poetiche degli altri autori a loro vicini. Consapevoli di contribuire, con il proprio operato, allo sviluppo della cultura nazionale, seppero scegliere il meglio della produzione internazionale, rielaborando, imitando, traducendo e interpretando. Conoscevano bene le esigenze intrinseche della cultura endogena, ma non erano disinformati neppure su quello che succedeva nel resto d'Europa per quanto riguarda la trasmissione del

\footnotetext{
${ }^{1}$ Cfr. AVIROVIĆ Lj., Le traduzioni della Divina Commedia in croato, in TOMASOVIĆ M. -AVIROVIĆ Lj., La divina traduzione. Tradurre in croato dall'italiano, EUT-Edizioni Università di Trieste, Trieste, 2012, p. 86: "La dualità di approccio traduttivo fu accesa tra i letterati croati. II dilemma persisteva a cominciare da san Girolamo e si protrasse fino al Rinascimento. I traduttori si chiedevano se era più opportuno tradurre "secondo le parole" (verbum de verbo), seguendo il ciceroniano ut interpres, oppure "secondo il senso", seguendo san Girolamo e il suo motto sensum de senso. Un fatto però rimarrà inconfutabile: tutti avevano assorbito la tesi ciceroniana che la traduzione letterale nasconde il significato del testo e che, seguendo san Girolamo, non era affatto importante esprimere parola con parola, bensì senso con senso».
} 
pensiero altrui, anzi, talvolta le loro traduzioni dal greco in latino, per esempio, risultarono utili anche ai traduttori italiani ${ }^{2}$.

Uno dei primi studiosi croati che si cimentarono nella traduzione delle opere di Dante, concedendo massima "aderenza" e "fedeltà" all'originale, fu Izidor Kršnjavi, poliedrica personalità di intellettuale (fu, tra l'altro, anche pittore, scrittore e professore presso la Cattedra di Storia dell'arte da lui stesso fondata presso l'Università di Zagabria nel 1878) che tanto improntò di sé la cultura croata dell'ultimo quarto del XIX secolo da ricevere moltissimi riconoscimenti per i propri contributi allo sviluppo della cultura e dell'arte croata e da far sì che il venticinquennio 1875-1900 fosse definito «l'era di Kršnjavi» ${ }^{3}$.

Ebbene, Kršnjavi non solo tradusse in prosa la Divina Commedia (accompagnandola con un commento - a giudizio dei competenti - esauriente ed interessante), ma sviluppò anche lo studio di Dante e ne introdusse le opere nei programmi universitari quale materia di studio obbligatoria per l'interpretazione della storia e della cultura proto-rinascimentali ${ }^{4}$.

Una parte del merito per la divulgazione della traduzione del capolavoro dantesco va, tra gli altri, anche a Walter Ljubibratić, professore di lingua italiana e tedesca al ginnasio di Zara, nel quale Kršnjavi trovò un valido collaboratore per la traduzione di un'opera così famosa ${ }^{5}$.

Dopo la traduzione di Kršnjavi, nel 1948 Mihovil Kombol tradusse con altrettanto successo le prime due cantiche della Divina Commedia ${ }^{6}$. Kombol fu uno storico e letterato croato che traspose in lingua croata opere dall'italiano e dal

2 Ivi, p. 97. In tal senso, nella postfazione alla Storia della letteratura croata (JELČIĆ D., Storia della letteratura croata (a cura di Ruggero Cattaneo), Guépard Noir, Milano, 2005, p. 518) pubblicata a Milano nel 2005, il curatore e traduttore Ruggero Cattaneo riporta una testimonianza eclatante: «[...] è significativo che Monti e Pindemonte potranno appoggiarsi anche alle traduzioni in esametri latini dell'lliade e dell'Odissea, condotte rispettivamente da Rajmund Kunić (1719-1794) e Berenard Zamanja (1735-1771)».

${ }^{3}$ MARUŠEVSKI O., U povodu Kršnjavijeva prijevoda "Božanstvene Komedije" (In riferimento alla versione croata della "Divina Commedia" ad opera di Izidor Kršnjavi), relazione presentata al Convegno internazionale Dante i slavenski svijet. Radovi međunarodnog simpozija * Dante e il mondo slavo. Atti del convegno internazionale (Dubrovnik, 26-29 ottobre 1981), Jugoslavenska akademija znanosti i umjetnosti, Zagreb, 1984, vol. 1, p. 383.

${ }^{4}$ Cfr. MARUŠEVSKI O., U povodu Kršnjavijeva prijevoda "Božanstvene Komedije", cit., pp. 383-399.

${ }^{5}$ Ivi, pp. 388-399.

${ }^{6}$ BRATULIĆ J., Jezik hrvatske književnosti u Kombolovu prijevodu "Božanstvene Komedije" (La tradizione del linguaggio letterario croato e la versione della "Divina Commedia" di Mihovil Kombol), relazione presentata al Convegno internazionale Dante $i$ slavenski svijet. Radovi međunarodnog simpozija * Dante e il mondo slavo. Atti del convegno internazionale (Dubrovnik, 26-29 ottobre 1981), Jugoslavenska akademija znanosti i umjetnosti, Zagreb, 1984, vol. 1, pp. 4956. 
tedesco. Lavorò come professore all'Accademia di Pedagogia e all'Accademia di Arti Drammatiche di Zagabria. Uno dei suoi maggiori interessi furono proprio le opere di Dante, tanto che cominciò a tradurre i primi canti dell'Inferno già nel 1928. Olinko Delorko ricorda che Kombol tradusse Dante con una metodologia particolare: alcuni canti li traduceva a memoria, cioè mentalmente, vale a dire senza trascriverli su carta finché non fosse arrivato alla versione definitiva. Riusciva in tal modo a tradurre mentre passeggiava, ad esempio, o mentre sorseggiava un caffè $o$ in qualunque altra circostanza in cui non fosse disturbato dalla presenza di altre persone. Allora, «un demone buono si impossessava della sua anima chiedendogli di vestire con i panni della nostra lingua, in modo energico e preciso, la potente parola poetica di Dante ${ }^{7}$. Josip Bratulić, per avvalorare la tesi delorkiana fondata sull'opinione che Kombol traducesse seguendo istintivamente la prima intuizione, riporta a titolo esemplificativo, alcuni passi del Purgatorio. Si veda, ad esempio, la prima redazione della traduzione di Purgatorio VI vv. $76-78^{8}$. Anche se vi è traccia di molti interventi correttivi nel complesso della traduzione di Kombol, secondo Bratulić la maggior parte delle soluzioni adottate, a livello di rima, nei primi tentativi traduttivi è rimasta inalterata nelle successive redazioni ${ }^{9}$.

Ah, ropska Italijo, zemljo jada,

lađo u buri a bez krmilara,

ne kraljice, već kućo bludna smrada ${ }^{10}$

Nella redazione finale, la medesima terzina subisce vistose modifiche nel primo e nel terzo verso, ma la rima è invariata:

O Italijo ropska, kućo jada, lađo u buri a bez krmilara, ne glavo naroda, već leglo smrada ${ }^{11}$

Ahi serva Italia, di dolore ostello, nave sanza nocchiere in gran tempesta, non donna di province, ma bordello! ${ }^{12}$

\footnotetext{
${ }^{7}$ Cfr. DELORKO O., O Mihovilu Kombolu povodom moje nadopune njegova prijevoda Danteova «Raja» in «Riječka revija», 9/1960, n. 5-6, pp. 271-277. (trad. it. di Eliana Moscarda Mirković).

${ }^{8}$ Ivi, pp. 53-54.

${ }^{9}$ BRATULIĆ J., Jezik hrvatske književnosti u Kombolovu prijevodu “Božanstvene Komedije”, cit., p. 53.

${ }^{10}$ Cfr. KOMBOL M. (a cura di), Primjeri iz stranih književnosti (Epika), St. Kugli, Zagreb, s.a. [ma 1935], p. 74.

${ }^{11}$ Cfr. ALIGHIERI D., Čistilište, preveo Mihovil Kombol, Matica hrvatska, Zagreb, 1955.

12 ALIGHIERI D., La Divina Commedia. Purgatorio, a cura di SAPEGNO N., La Nuova Italia, Firenze $1985^{3}\left(1956^{1}\right)$, pp. 64-65.
} 
Lo stesso vale per l'ultima terzina del Purgatorio, che è stata tradotta prima del 1935, quindi più di dieci anni prima del completamento della traduzione della cantica. Ebbene, secondo Bratulić, nonostante la dovuta revisione dei versi, l'atmosfera di fondo komboliana resta immutata nella redazione del 1955:

Od svetog vala preporođen sinem

ko mlade biljke obnovljene lišćem,

čist i do zvijezda spreman da se vinem. ${ }^{13}$

lo ritornai da la santissima onda

rifatto sì come piante novelle

rinovellate di novella fronda,

puro e disposto a salire a le stelle. ${ }^{14}$

Mihovil Kombol dedicò quasi la totalità del proprio tempo alla traduzione di quest'opera, perché sapeva che la precisione e l'accuratezza della propria attività avrebbero potuto costituire un importante stimolo allo sviluppo della lingua e della letteratura croate ${ }^{15}$. II processo di traduzione dell'intero poema dantesco, proprio a causa di tale cura meticolosa, durò così a lungo che a Kombol non fu purtroppo possibile condurlo a termine: egli morì infatti nel 1955, prima di poter finire la cantica del Paradiso (che venne, infatti, completata in un secondo momento, da Olinko Delorko).

Molti critici e letterati hanno sostenuto l'opinione che la traduzione di Kombol possa affrontare senza soggezioni il paragone con l'originale, anche perché le traduzioni komboliane non furono realizzate secondo quella che fino a quel tempo veniva considerata l' "unità testuale minima" nell'ambito della traduzione di testi poetici (cioè, il verso), ma lo studioso tradusse l'intera Comedia in terzine. Le terzine, infatti, «rispettano la rima e l'endecasillabo, motivo in più che ha reso la suddetta traduzione sempre più apprezzata nel tempo, facendola considerare come la più accurata» ${ }^{16}$. Kombol, nel tradurre i versi di Dante, ebbe come punto di riferimento il canto completo, ma pose particolare attenzione alla terzina: perciò la scelta della parola centrale, della parola-guida nella costruzione delle soluzioni

\footnotetext{
${ }^{13}$ Cfr. KOMBOL M. (a cura di), Primjeri iz stranih književnosti (Epika), St. Kugli, Zagreb, s.a. [ma 1935], cit., p. 74.

Cfr. ALIGHIERI D., Čistilište, preveo Mihovil Kombol, cit., p. 203 ad I: «Ko, mlade biljke lišćem hvoja novih / podmlađene iz rijeke gdjeno jezde / presveti vali obnovljen isplovih, / čist i za uzlet spreman među zvijezde //».

${ }^{14}$ ALIGHIERI D., La Divina Commedia. Purgatorio, a cura di SAPEGNO N., cit., p. 376.

${ }^{15}$ BRATULIĆ J., Jezik hrvatske književnosti u Kombolovu prijevodu "Božanstvene Komedije", cit., p. 49.

${ }^{16}$ Ivi, pp. 49-50.
} 
versificatorie, fu dettata dalla rima (a livello della terzina o delle terzine). La scelta del lessema successivo ricadeva invece su quello avente un'accentazione sulla quarta sillaba.

Secondo Josip Jernej, però, anche se questa versione può essere considerata corretta e fedele, linguisticamente trasparente e metricamente curata, rimane priva nel suo complesso del necessario afflato poetico ${ }^{17}$. Si tratta forse di un giudizio un po' troppo severo, se si considera che la lingua di Kombol ha agito da modello non solo per le traduzioni in croato della poesia italiana antica, bensì anche per la poesia originale croata moderna. Se dunque, all'apparizione della traduzione dell'Inferno, parte della critica rimproverò a Kombol l'arcaicità dell'espressione, è pur vero che lo stile komboliano è diventato, in Croazia, parte costitutiva dell'espressione di tutta una generazione poetica.

Esiste invece del resto tutta una serie di traduzioni che effettivamente non sono riuscite a "tenere il passo" con il livello espressivo di Dante. Una di queste, manifestamente criticata da Antun Djamić, perché ha addirittura alterato il significato di certe parole e concetti, è quella di Stjepan Markuš.

La traduzione della Divina Commedia di Stjepan Markuš presenta in realtà, secondo il Djamić, moltissimi errori, non solo di tipo grammaticale e sintattico, ma anche di carattere etimologico e semantico ${ }^{18}$. Basti dire che molte parole, di facies apparentemente croata, utilizzate dal Markuš sono state persino definite «inesistenti ed inventate» ${ }^{19}$. Alcuni lessemi da lui usati, insomma, non appartengono alla lingua croata.

17 JERNEJ J., Appunti su una recente versione croata della "Divina Commedia", in "Studia Romanica et Anglica Zagrabiensia», n. 29-30, Zagreb, 1970-1971, pp. 565-566.

${ }^{18}$ Antun Djamić nell'esaustivo saggio Hrvatski jezik u Markuševu prijevodu Božanstvene komedije rimprovera a Markuš l'utilizzo, nella sua traduzione, di grafie erronee e di forme inesatte di alcuni nomi stranieri, l'errato uso dei preteriti, l'uso arbitrario delle maiuscole, la scarsa coerenza nell'assimilazione delle consonanti o la loro omissione, l'errata accentuazione dell'endecasillabo, le rime imperfette, gli errori di grammatica, l'uso di parole sconosciute o di quelle non appartenenti alla lingua letteraria croata. (Djamić A., Relazione presentata al Convegno internazionale Dante i slavenski svijet. Radovi međunarodnog simpozija * Dante e il mondo slavo. Atti del Convegno internazionale, cit., pp.109-136).

In questa sede, per motivi di spazio, riportiamo solo un paio di esempi tratti dalla vasta campionatura esemplificativa di Djamić. Nel verso «tastom sve će mlade zvati» (Paradiso, XXVI, v. 93: "a cui ciascuna sposa è figlia e nuro»), secondo il critico, Markuš afferma che le "giovani donne" chiameranno qualcuno tast. Nella lingua croata il verso non può trovare riscontro perché con tast si designa il suocero da parte del marito, mentre per indicare il suocero da parte della moglie si usa il termine svekar. Djamić attribuisce al traduttore una imperfetta conoscenza dei termini indicanti i gradi di parentela, che deriva probabilmente dalla sua appartenenza linguistica alla variante sottodialettale caicavica della lingua croata e che usa il termine tast in modo indifferenziato. 
A livello metrico, Djamić individua altresì un'erronea accentuazione dell'endecasillabo. Una tale tipologia di errore è evidente, ad esempio, in alcuni versi del canto XXI dell'Inferno. Gli accenti costituiscono l'elemento di base della versificazione dantesca, mentre nei vv. 43-48 possiamo vedere come gli accenti sugli endecasillabi non corrispondono agli accenti esatti della lingua croata.

Niz tvŕdu hríd on, nákon štó ga sjúri, pohŕli; pás , kad, púšten s lánca, góni za tátom, táko níkad bŕz ne žúri.

Utónu táj, no s léđimá izróni tad gráknu, móst pod sóbom štó ih skríva, «Tu Svéta Líca néma!» ná nj demóni. ${ }^{20}$

Là giù 'I buttò, e per lo scoglio duro si volse; e mai non fu mastino sciolto 45 con tanta fretta a seguitar lo furo.

Quel s'attuffò, e tornò su convolto, ma i demon che del ponte avean coperchio, gridar: «Qui non ha loco il Santo Volto»: ${ }^{21}$

In queste due terzine, spiega Djamić, gli accenti tonici conformi alla lingua croata standard sono stati da lui segnalati ponendo in corsivo le vocali su cui poggiano, mentre appartengono allo schema metrico dell'endecasillabo quelli indicati col segno grafico dell'accento acuto. Dal confronto si vede chiaramente come gli accenti dello schema dell'endecasillabo non coincidano con quelli della lingua croata standard in due punti nei vv. 43, 45 e 47; in un punto nei vv. 44 e 48; in ben quattro punti, infine, nel v. 46 .

Nel verso «[duši] Inferna svakoj pripadnosti odredi» (Inferno, V, v. 10: «vede qual luogo d'inferno è da essa»). In questo caso Djamić si chiede se con il sostantivo pripadnosti ('appartenenza') Markuš rispetti a pieno la funzione che caratterizza il giudice infernale dantesco. Minosse, infatti, non giudica l'appartenenza delle anime all'Inferno (è ovvio che vi appartengano), ma indica il luogo in cui verranno puniti i peccatori.

${ }^{19}$ Ad esempio; Antoniana (Paradiso, XXIX, v. 124), Currada (Paradiso, XV, v. 139), Hug (Purgatorio, $X X$, v. 49), poieii (Purgatorio, XXVII, v. 56).

${ }^{20}$ ALIGHIERI D., Inferno. Complementi, preveo i prikazao Stjepan Markuš, vlast.nakl., Zagreb, 1968.

${ }^{21}$ ALIGHIERI D., La Divina Commedia, cit., p. 239. 
Sebbene il ritmo markušano, misurato in base ai parametri dell'accentazione letteraria standard della lingua croata, non sia perfetto, Djamić riconosce comunque al traduttore il merito di essersi avvicinato alla perfezione. ${ }^{22}$

Tutti questi tentativi di traduzione, con esiti - come s'è avuto modo di osservare - ora più ora meno felici, hanno comunque fatto sì che la lingua croata, portata a misurarsi con la complessità linguistica del capolavoro dantesco, arricchisse il proprio vocabolario, e rendendo omaggio ai grandi classici della letteratura, potenziasse le proprie possibilità espressive. II contributo di queste traduzioni consiste dunque nell'aver dato prova che, la lingua croata, dal momento in cui è diventata lingua nazionale (nell'ambito della Rinascita Nazionale Croata, 1830-1874), si è evoluta tanto da poter reggere il paragone con l'innovazione linguistica operata nel Trecento da Dante Alighieri.

Il debito della cultura croata nei confronti di Dante data, del resto, da lungo tempo, ed in tal senso non sembra affatto casuale che lo sforzo di tradurre la sua opera nella nostra lingua si perpetua dal risorgimento nazionale e linguistico, ossia dalla prima traduzione apparsa in "Zori Dalmatinskoj" nel 1845, fino alla traduzione di Kombol, cioè da almeno un secolo. ${ }^{23}$

\section{L’influsso della Comedìa su Petar Zoranić}

Abbiamo già notato come un gran numero di autori croati abbia utilizzato $i$ testi classici internazionali come base per sviluppare il proprio stile scrittorio. Ciò che non è sin qui ancora emerso, e di cui si farà parola qui di seguito, è l'analogia esistente, a livello macro-tematico, tra la Divina Commedia di Dante Alighieri ed il primo romanzo della letteratura croata - Planine ("Montagne") di Petar Zoranić (Zara 1508-post 1569), uno dei più famosi esponenti del Rinascimento croato.

Planine è prova evidente dell'importanza dell'influsso di Dante Alighieri sulla letteratura croata. Questo romanzo è stato infatti scritto sul modello della Divina Commedia ed è diventato poi la base per la stesura, in epoche successive, di altri

\footnotetext{
${ }^{22}$ «O tí, kog úda króz Inférno spjéše, / prepóznaj méne, jé l’ ti dáno bíva; / još já bjeh žív kad tí već djéčak bjéše» // A já ću njému: «Pátnja bít će kríva / Iz móje dá te móžda tjéra svijésti, / te kánda níkad né vidjéh te žíva. (Inferno VI vv. 40-45). - In questo brano tutti gli accenti coincidono nei versi 41 e 43; mentre non coincidono in un punto nel verso 44 , in due punti nel verso 45 ed in tre punti nei versi 40 e 42.

${ }^{23}$ BRATULIĆ J., Jezik hrvatske književnosti u Kombolovu prijevodu "Božanstvene Komedije", cit., p. 49 (trad. it. di Eliana Moscarda Mirković).

${ }^{23}$ Ivi, pp. 53-54.
} 
romanzi croati $^{24}$. Nella trama di Planine, quando il pastore Zoran - che ne è il protagonista - parte per il proprio viaggio allegorico, si coglie subito l'analogia con la partenza di Dante-personaggio per le vie dell'Inferno, del Purgatorio e del Paradiso. Anche Mihovil Kombol nota delle somiglianze tra le due opere, evidenti soprattutto quando Zoran incontra la bestia dalle sette teste (simbolo dei sette peccati capitali) e si trova davanti alla porta del diavolo. Tutti elementi che sono in stretta corrispondenza con l'Inferno di Dante. Kombol sostiene anche che, come diversi scrittori hanno influito sulla scrittura di Zoranić, così senza dubbio alcuni degli ipotesti presenti in Planine appartengono alla produzione letteraria dantesca ${ }^{25}$.

Vari critici letterari hanno provato a spiegare come la genialità di Dante abbia influito sul romanzo di Zoranić e la conclusione principale è che l'allegoria sia l'elemento principale che connette le due opere. Questa allegoria viaggia per mondi magici e inesistenti, per arrivare, come afferma V. Štifanić, ad un unico approdo: "La grazia divina conduce l'umano tra il male terrestre per arrivare fino alla conoscenza della verità di Dio» ${ }^{26}$. La finalità del viaggio del pastore di Zoranić è identica a quella del viaggio di Dante: ritrovare la verità in questo mondo pieno di perfidia e malizia e ritrovare il messaggio etico e morale dell'umanità.

\section{La presenza di Dante nelle opere di Andrija Kačić Miošić}

Le opere di Dante Alighieri hanno influito su diversi ambiti particolari della storia letteraria croata. Non stupisce, perciò, il fatto che proprio dell'Inferno di Dante si sia trovata traccia anche nei testi teologici croati.

Nonostante il successo e l'influenza dei testi danteschi su molte opere croate, a partire dall'epoca rinascimentale e fino al XVIII secolo si riscontra una sorta di stasi nello studio delle opere di Dante ${ }^{27}$, e non solo in Croazia ma anche, più in generale, a livello europeo. I cambiamenti subentrati nella sfera culturale e sociale fecero insomma sì che le porte della letteratura croata, fino ad allora aperte all'influenza di Dante, si chiudessero per un certo periodo di tempo

\footnotetext{
${ }^{24}$ FALIŠEVAC D., Odjeci Danteova djela u Zoranićevim "Planinama" (Echi di Dante nelle "Montagne" di Petar Zoranić), relazione presentata al Convegno internazionale Dante i slavenski svijet. Radovi međunarodnog simpozija * Dante e il mondo slavo. Atti del Convegno internazionale (Dubrovnik, 26-29 ottobre 1981), Jugoslavenska akademija znanosti i umjetnosti, Zagreb, 1984, vol. 1, p. 161.

${ }^{25}$ KOMBOL M., Povijest hrvatske književnosti do preporoda, Matica Hrvatska, Zagreb, 1961², p. 140.

${ }^{26}$ FALIŠEVAC D., Odjeci Danteova djela u Zoranićevim "Planinama", cit., p. 165.

${ }^{27}$ PERILLO F. S., Andrija Kačić Miošić e Dante, relazione al Convegno internazionale Dante i slavenski svijet. Radovi međunarodnog simpozija * Dante e il mondo slavo. Atti del convegno internazionale (Dubrovnik, 26-29 ottobre 1981), Jugoslavenska akademija znanosti i umjetnosti, Zagreb, 1984, vol .1, p. 453.
} 
all'ascendente del grande Fiorentino. In questo contesto un po' nebuloso ed oscuro, Dante tornò a trovare un proprio posto nelle opere di Andrija Kačić Miošić, un monaco e scrittore croato del XVIII secolo che dedicò la propria vita alla religione e alla scrittura, consacrandosi alla stesura di molte opere in latino e in volgare, e che raggiunse la fama nel panorama letterario croato con il libro in versi $\mathrm{e}$ in prosa intitolato Razgovor ugodni naroda slovinskoga (Dilettevole conversazione del popolo slavo) e con la cronaca Korabljica Svetoga pisma (Arca della Sacra Scrittura) ${ }^{28}$.

E proprio in queste due opere possiamo trovare elementi riconducibili a Dante. Importante è notare come Miošić si sia ispirato moltissimo all'illuminista Filip Grabovac e, anche se egli non lo dice in maniera esplicita, si vede nei suoi scritti un forte influsso di Dante, quale rappresentante di un'attività letteraria preumanista.

Kačić Miošić tenne inoltre per dieci anni l'incarico di lector generalis nello Studio teologico aperto ai membri dell'ordine francescano e del clero secolare di Šibenik (Sebenico). Non sembra quindi una coincidenza che nella biblioteca francescana della città si conservi una descrizione della cantica dell'Inferno risalente al XV secolo ${ }^{29}$. La cosa interessante è che «l'anonimo autore si sofferma sui nove giorni di dantesca memoria con una lettura strumentale dell'Inferno, destinata a influire sulla coscienza dei fedeli in un'epoca in cui più rigorosa si voleva l'applicazione dei precetti cristiani e più urgenti si conclamavano le istanze di ravvedimento e di ritorno alla fede antica» ${ }^{30}$.

Nelle due opere di Kačić Miošić non si menziona solo Dante: nel primo libro della Dilettevole conversazione del popolo slavo si accenna infatti al padre della lingua italiana insieme ai grandi nomi della storia, quali Gregorio Magno, Innocenzo III e Benedetto da Norcia. In Pisma od kuće Frankopanovića, in cui lo scrittore celebra la ben nota casata feudale dei Frankopani, anche Dante trova posto nei versi riportati qui di seguito:

\footnotetext{
${ }^{28}$ Cfr. Wikipedia, s.v. Andrija Kačić Miošić (http://hr.wikipedia.org/wiki/Andrija_Kačić_Miošić), pagina consultata il 14 maggio 2013)

${ }^{29}$ PERILLO F. S., Andrija Kačić Miošić e Dante, cit., p. 453.

${ }^{30}$ Ibidem.
} 


\begin{abstract}
Po svem svitu Dante glasoviti, ù nauku puno ponositi, Fiorentin koji se zoviše, od kolina Frankopana biše, a od kuće na glasu viteza Aldigera, velikoga kneza od bijela Fiorence grada, kojano se nalazi i sada. ${ }^{31}$
\end{abstract}

Da questi versi si vede chiaramente che Miošić riteneva che Dante fosse un antenato dei Frankopani. Anni dopo, precisamente nell'anno 1845, tale teoria sarebbe stata confutata da Donato Fabianich, che ricondusse la genealogia degli Alighieri a Firenze, dichiarando la loro appartenenza alla stirpe degli Elisei e non ai Frankopani. Rimane nondimeno il fatto che in territorio croato, anche se per breve durata, Dante fu associato alla stirpe feudale frankopana ${ }^{32}$.

\title{
4. Il modello di Dante in Tin Ujević
}

Si è visto che le opere di Dante Alighieri, soprattutto la Divina Commedia, sono state fonte d'ispirazione di molti famosi scrittori croati. Božidar Petrač sostiene addirittura che scrittori come Marko Marulić (Marco Marulo) abbiano preso Dante come modello per scrivere le proprie opere ${ }^{33}$.

Non sorprende pertanto il fatto che anche uno scrittore come Tin Ujević, che ha fatto la storia della letteratura croata, abbia scelto l'eccellenza Dante come fonte d'ispirazione. La cosa interessante è che, nella ricezione dell'opera dantesca da parte di Ujević, come modello di riferimento viene assunta la Vita nova e non la Divina Commedia, com'era stato per la maggior parte degli altri scrittori. Petrač sostiene che il periodo durante il quale, in Ujević, più forte fu l'influsso di Dante Alighieri sia quello compreso tra il 1920 ed il 1926. In questo periodo, in effetti, Ujević compose le proprie opere più famose: nel 1920 diede alle stampe la raccolta di poesie Lelek sebra (Il pianto dello schiavo), e ancora nel 1926 pubblicò un'altra

${ }^{31}$ LUUBIĆ Š., Commissiones et Relationes Venetae, III, (Monumenta spectantia historiam Slavorum meridionalium, XI), JAZU, Zagreb, 1880, p. 126, nota 1: «Per tutto il mondo Dante famoso, / di scienza grandemente fiero, / che si nomava Fiorentino, / della stirpe dei Frankopani era, / e del casato del cavaliere di fama / Aldighieri, gran principe / della bianca città di Fiorenza / che esiste ancora.».

32 PERILLO F. S., Andrija Kačić Miošić e Dante, cit., p. 454-461.

33 PETRAČ B., Dante nell'opera di Tin Ujević (1891-1955), relazione presentata al Convegno internazionale Dante i slavenski svijet. Radovi međunarodnog simpozija - Dante e il mondo slavo. Atti del convegno internazionale, (Dubrovnik, 26-29 ottobre 1981), Jugoslavenska akademija znanosti i umjetnosti, Zagreb, 1984, vol. 2, pp. 471-478. 
raccolta di poesie, intitolata Kolajna (Collana). Queste due opere sono inoltre in stretta connessione con le prose poetiche di tipo confessionale intitolate EI sentimiento trágico de la vida, del 1922, Trubadur u Provansi (II trovatore in Provenza), sempre del 1922, e Ispit savjesti (L'esame di coscienza), del 1923. Questi scritti sono collegati l'uno all'altro perché, come i grandi poeti del passato erano soliti commentare la loro produzione lirica, fornendo informazioni sul proprio modo di scrivere ed esplicitando la propria poetica, così anche Tin Ujević volle connettere le proprie opere in modo da poterle spiegare una attraverso I'altra ${ }^{34}$. Come dunque Dante, per l'appunto nella Vita nova, aveva commentato nelle parti in prosa le liriche scritte per Beatrice e colà raccolte, così anche Ujević, in un misto di prosa e poesia, commenta le proprie liriche per avvicinarle ai lettori, ottenendo un prosimetro unico nel suo genere.

Anche se, in un certo senso, Tin Ujević fa di Dante un proprio modello, è pur vero che egli gli antepone sia Petrarca sia i Trovatori. Ciò si spiega facilmente col fatto che la poetica moderna del XIX secolo è molto più vicina, tematicamente e strutturalmente, al petrarchismo piuttosto che al divino Poeta. Nonostante ciò, Ujević amò molto anche il modo di scrivere di Dante e fece buon uso del suo esempio, come spiega Petrač. Questo buon uso è visibile, ad esempio, in Serenità (Vedrina), poesia che ha come tema l'immensità dell'Universo e che in modo trasparente rimanda al famoso brano sull'armonia delle sfere del I canto del Paradiso:
[...]
Pružite uho, čujte: misli zvuče, sviraju mašte kao zvonke sfere, a kolo zvijezda bijelo srce vuče svemirom gdje se oblak jave stere. Gluma je rajska, da sam čak i Titan: svijet tako golem, a ja tako sitan ${ }^{35}$
Ascoltate, sentite: pensieri risuonano, sonan fantasie come sfere sonore, e la danza degli astri trascina il bianco cuore per il cosmo dove s'estende la nube del dormiveglia. È il Paradiso, fossi anche Titano: il mondo così grande, ed io così piccino ${ }^{36}$

\footnotetext{
${ }^{34}$ Ibidem.

35 UJEVIĆ T., Vedrina, in Odabrana djela Tina Ujevića, Knjiga prva, August Cesarec, Zagreb, 1979, p. 72.
} 
Nonostante l'influenza di Dante Alighieri, ad ogni modo, Tin Ujević creò piuttosto una sorta di "petrarchismo ujevichano" (come i suoi discepoli definiscono il suo stile), cambiando la percezione della poetica, ossia trasformando la «mistica della passione» in una "passione per la mistica» ed aprendo in tal modo le porte ad una nuova era della poesia romantica croata.

\section{Alcuni interpreti dalmati della Divina Commedia}

I testi di Dante Alighieri non sono stati usati solo come spunto per sviluppare la letteratura croata, ma sono anche diventati oggetto di studio da parte della critica letteraria croata, che ha così inteso offrire una nuova strada nell'avviamento allo studio delle opere di Dante.

Numerosi sono i critici croati che hanno chiosato ed interpretato le opere di Dante, ma i commenti che più di altri hanno segnato la storia letteraria croata sono sicuramente quelli di Niccolò Tommaseo e di Antonio Lubin ${ }^{37}$.

Niccolò Tommaseo, nelle vesti di filologo e lessicografo, ha analizzato soprattutto la parte funzionale dello stile e della lingua di Dante, facendo posto anche ad un'analisi psicologica della Divina Commedia. La novità della critica di Tommaseo sta proprio nell'approccio psicologico al testo, perché prima di lui questa dimensione non era stata troppo discussa né presa in considerazione. Quello che secondo altri critici è stato, invece, trascurato nel suo commento, è la parte estetica, come pure l'analisi politica, etica e filosofica. II Tommaseo è stato inoltre criticato per gli errori di grafia, le citazioni imprecise, le eccessive annotazioni, ma soprattutto la troppa moralizzazione ${ }^{38}$.

Nonostante le critiche che gli sono state mosse, il Tommaseo ha dato un grande contributo alla scoperta di nuovi orizzonti riguardanti Dante Alighieri e la sua opera somma, la Divina Commedia. La Divina Commedia accompagnata dal commento di Niccolò Tommaseo è sicuramente uno dei volumi che non devono mancare dagli scaffali di ogni dantista che si rispetti.

Antonio Lubin (Ante Lubin, Traù 1809-1900), altro grande dantista della Dalmazia, considerava inaccettabile un'interpretazione soggettiva e pragmatica del testo dantesco, ritenendo piuttosto che una buona esegesi di esso non dovesse

\footnotetext{
${ }^{36}$ PETRAČ B., Dante nell'opera di Tin Ujević (1891-1955), cit., pp. 471-478.

${ }^{37}$ RABAC-ČONDRIĆ G., Alcune interpretazioni dantesche di autori dalmati, relazione presentata al Convegno internazionale Dante i slavenski svijet. Radovi međunarodnog simpozija-Dante e il mondo slavo: atti del convegno internazionale (Dubrovnik, 26-29 ottobre 1981), Jugoslavenska akademija znanosti i umjetnosti, Zagreb, 1984, vol. 2, pp. 537-544.

38 Ibidem.
} 
distanziarsi dal pensiero politico e religioso di Dante Alighieri. Lubin, come Niccolò Tommaseo, è stato però criticato per un numero troppo esteso di commenti, note, illustrazioni, che secondo alcuni critici appesantiscono il testo letterario.

Anche se la critica letteraria non sempre ha apprezzato il lavoro dei due dantisti dalmati, ogni interpretazione, ogni opera scritta sul modello della Divina Commedia o di qualunque altra opera di Dante Alighieri, ha sicuramente contribuito all'evoluzione della letteratura e della lingua croata.

\section{Conclusione}

In questo saggio si è visto che Dante Alighieri, fungendo da modello artistico ed espressivo ai grandi scrittori della letteratura croata, ha fornito un rilevante contributo indiretto allo sviluppo della stessa lingua croata. Le traduzioni delle sue opere, soprattutto della Divina Commedia, hanno arricchito la letteratura croata ed hanno in tal modo permesso ai nostri scrittori di evolversi artisticamente. In queste traduzioni sono venute a contatto due lingue, una romanza e l'altra slava, che pur rappresentando culture diverse mostrano al tempo stesso anche affinità tipologiche e culturali essendo entrambe, come ben nota J. Jernej, lingue mediterranee. Ciò che è stato possibile valorizzare, grazie a tali traduzioni, è la ricchezza e varietà espressiva della lingua nazionale croata. Le traduzioni realizzate dai vari autori che abbiamo menzionato hanno, infatti, permesso a quest'ultima di affermarsi nell'ambito della letteratura mondiale. Anche grazie ad esse, in altri termini, la lingua croata ha potuto dar prova del proprio valore e della propria maturità espressiva, nell'affrontare una sfida ardua come quella della traduzione della Comedia. Non è quindi eccessivo sostenere che Dante Alighieri ha dato, quantomeno per via indiretta, un contributo notevolissimo allo sviluppo del patrimonio letterario e linguistico croato. 


\section{BIBLIOGRAFIA}

ALIGHIERI, D., 1955, Čistilište, preveo Mihovil Kombol, Matica hrvatska, Zagreb.

ALIGHIERI, D., 1968, Inferno. Complementi, preveo i prikazao Stjepan Markuš, vlast. nakl., Zagreb.

ALIGHIERI, D., $1985^{3}$ (1956¹), La Divina Commedia. Purgatorio, a cura di SAPEGNO N., La Nuova Italia, Firenze.

ALIGHIERI, D., 2004, Božanstvena komedija, preveli s talijanskog Mihovil Kombol i Mate Maras (Raj XVIII-XXXIII), Globus Media, Zagreb.

AVIROVIĆ, Lj., 2012, Le traduzioni della Divina Commedia in croato, in TOMASOVIĆ M. AVIROVIĆ Lj., La divina traduzione. Tradurre in croato dall'italiano, EUT-Edizioni Università di Trieste, Trieste.

BRATULIĆ, J., 1984, Jezik hrvatske književnosti u Kombolovu prijevodu "Božanstvene Komedije" (La tradizione del linguaggio letterario croato e la versione della "Divina Commedia", fatta da Mihovil Kombol), relazione presentata al Convegno internazionale Dante i slavenski svijet. Radovi međunarodnog simpozija * Dante e il mondo slavo. Atti del convegno internazionale (Dubrovnik, 26-29 ottobre 1981), Jugoslavenska akademija znanosti i umjetnosti, Zagreb, vol. 1, pp. 49-56.

DELORKO, O., 1960, O Mihovilu Kombolu povodom moje nadopune njegova prijevoda Danteova «Raja» in «Riječka revija», 9/1960, n. 5-6, pp. 271-277.

DJAMIĆ, A., 1984, Hrvatski jezik u Markuševu prijevodu "Božanstvene Komedije" (La lingua croata della versione della "Divina Commedia" fatta da S.Markuš), relazione presentata al Convegno internazionale Dante $i$ slavenski svijet. Radovi međunarodnog simpozija * Dante e il mondo slavo. Atti del convegno internazionale (Dubrovnik, 26-29 ottobre 1981), Jugoslavenska akademija znanosti i umjetnosti, Zagreb, vol. 1, pp. 109-136.

FALIŠEVAC, D., 1984, Odjeci Danteova djela u Zoranićevim "Planinama" (Echi di Dante nelle "Montagne" di Petar Zoranić), relazione presentata al Convegno internazionale Dante i slavenski svijet. Radovi međunarodnog simpozija* Dante e il mondo slavo. Atti del convegno internazionale (Dubrovnik, 26-29 ottobre 1981), Jugoslavenska akademija znanosti i umjetnosti, Zagreb, vol. 1, pp. 161-173.

JELČIĆ, D., 2005, Storia della letteratura croata (a cura di Ruggero Cattaneo), Guépard Noir, Milano.

JERNEJ, J., 1970-1971, Appunti su una recente versione croata della "Divina Commedia", in «Studia Romanica et Anglica Zagrabiensia», n. 29-32, Zagreb, pp. 565-590.

KOMBOL, M., (a cura di), [1935], Primjeri iz stranih književnosti (Epika), St. Kugli, Zagreb, s.a. [ma 1935].

LJUBIĆ Š., 1880, Commissiones et Relationes Venetae, III (Monumenta spectantia historiam Slavorum meridionalium, XI), Jugoslavenska akademija znanosti i umjetnosti, Zagreb. 
MARUŠEVSKI, O., 1984, U povodu Kršnjavijeva prijevoda "Božanstvene Komedije" (In riferimento alla versione croata della "Divina Commedia" ad opera di Izidor Kršnjavi), relazione presentata al Convegno internazionale Dante $i$ slavenski svijet. Radovi međunarodnog simpozija * Dante e il mondo slavo. Atti del convegno internazionale (Dubrovnik, 26-29 ottobre 1981), Jugoslavenska akademija znanosti i umjetnosti, Zagreb, vol. 1, pp. 383-399.

PERILLO, F. S., 1984, Andrija Kačić Miošić e Dante, relazione presentata al Convegno internazionale Dante i slavenski svijet. Radovi međunarodnog simpozija * Dante e il mondo slavo. Atti del convegno internazionale (Dubrovnik, 26-29 ottobre 1981), Jugoslavenska akademija znanosti i umjetnosti, Zagreb, vol. 1, pp. 453-462.

PETRAČ, B., 1984, Dante nell'opera di Tin Ujević (1891-1955), relazione presentata al Convegno internazionale Dante i slavenski svijet. Radovi međunarodnog simpozijaDante e il mondo slavo. Atti del convegno internazionale (Dubrovnik, 26-29 ottobre 1981), Jugoslavenska akademija znanosti i umjetnosti, Zagreb, vol. 2, pp. 471-478.

RABAC-ČONDRIĆ, G., 1984, Alcune interpretazioni dantesche di autori dalmati, relazione presentata al Convegno internazionale Dante $i$ slavenski svijet. Radovi međunarodnog simpozija * Dante e il mondo slavo. Atti del convegno internazionale (Dubrovnik, 26-29 ottobre 1981), Jugoslavenska akademija znanosti i umjetnosti, Zagreb, vol. 2, pp. 537-544.

UJEVIĆ, T., 1979, Odabrana djela Tina Ujevića, Knjiga prva, August Cesarec, Zagreb.

\section{SITOGRAFIA}

Wikipedia, s. v. Andrija Kačić Miošić (http://hr.wikipedia.org/wiki/Andrija_Kačić _Miošić), pagina consultata il 14 maggio 2013) 


\section{UTJECAJ DJELA DANTEA ALIGHIERIJA NA HRVATSKU KNJIŽEVNOST I KULTURU}

Ovaj rad predstavlja književno istraživanje o kulturnom utjecaju Dantea Alighierija na hrvatsku književnost. Namjera je čitateljima približiti važnost utjecaja Dantea Aligherija u Hrvatskoj jer mnogi zanemaruju činjenicu da je ovaj velikan boravio na našim prostorima i da ih je, zadivljenim njima, opisao u Božanstvenoj komediji. Dante Alighieri nije samo omogućio razvoj talijanskoga jezika, već otkrivamo da je i hrvatskom jeziku dao značajan poticaj, a kroz prijevode Kombola i Kršnjavija, pridonio otvaranju puta Hrvatske k europskom književnom vrhu.

Ključne riječi: Dante Alighieri, Božanstvena komedija, hrvatska književnost, Kombol, Kršnjavi, Markuš

\section{THE CULTURAL INFLUENCE OF DANTE'S LITERARY WORKS ON CROATIAN LITERATURE}

The paper includes a research regarding the cultural influence of Dante Alighieri literary production on Croatian literature. The main purpose of this work is to bring a closer view of the influence of Dante Alighieri to Croatian readers because a lot of people may ignore his stay in Croatia and the importance of his main work - The Divine Comedy in our country. The paper analyses the influence on local writers and also on the translators of his works. Dante Alighieri has facilitated not only the development of the Italian language, but also of the Croatian language, especially with the translations of Kombol and Kršnjavi.

Key words: Dante Alighieri, Divine Comedy, Croatian Literature, Kombol, Markuš 